\title{
Drahtloses und bildgebendes Multi-Sensorsystem integriert in eine Handgelenksorthese für ein nichtinvasives Langzeitmonitoring
}

\author{
M. Schimmack ${ }^{1)}$, R. Sukhikh ${ }^{1)}$, Prof. Dr. A. Georgiadis ${ }^{1}$, Prof. Dr. C.-H. Siemsen ${ }^{2)}$ \\ 1)Leuphana Universität Lüneburg, Institut für Produkt- und Prozessinnovation \\ Volgershall 1, 21339 Lüneburg, Deutschland \\ ${ }^{2)}$ Hochschule für Angewandte Wissenschaften Hamburg, Fakultät Life Sciences \\ Lohbrügger Kirchstraße 65, 21033 Hamburg, Deutschland \\ schimmack@uni-leuphana.de | ruslan.suhih@stud.leuphana.de | georgiadis@uni-leuphana.de | claus.siemsen@haw-hamburg.de
}

\begin{abstract}
Entwicklung eines bildgebenden, textilbasierten Mikrosystems, das in eine gipsstabile Handgelenksorthese integriert ist, um eine in situ Langzeitüberwachung während der Heilungsphase von Unterarmfrakturen zu ermöglichen. Ein Multi-Sensorsystem soll frühzeitig bekannte Entzündungsindikatoren wie Rötungen, Überwärmungen, Schwellungen oder Durchblutungsstörungen messtechnisch erfassen und durch eine Embedded-Mikroelektronik ausgewertet und gemeldet werden. Trophische Erkrankungen wie z.B. beim komplexen regionalen Schmerzsyndrom (CRPS), die sich auf Grund einer Fraktur ergeben können, sollen durch eine Langzeitthermographie registriert werden, um zeitnah Gegenmaßnahmen ergreifen zu können. Eine sichere, interoperable und gleichzeitige Kommunikation von mehreren Sensoren steht im Vordergrund, um den komplexen Anforderungen an das integrierte Gesamtsystem gerecht zu werden. Der Datenaustausch und die Programmierung des Embedded-Systems basiert auf einer weltweit standardisierten, drahtlosen Übertragung und ermöglicht
\end{abstract}

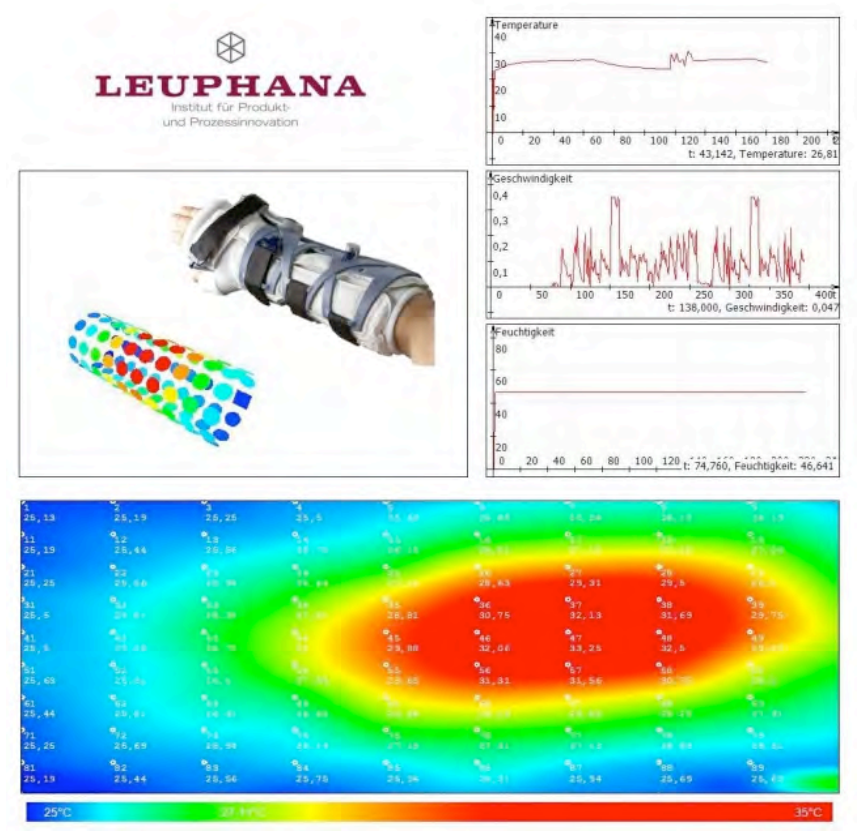

Abb. 1: Darstellung der Auswerte- und Visualisierungssoftware für Diagnostik, Therapie und Monitoring

die erfassten Messdaten an eine mobile, medizinische Workstation zu schicken. Wie in Abbildung 1 dargestellt, kann mit Hilfe einer eigens entwickelten Auswerte- und Visualisierungssoftware die auf dem Embedded-System gespeicherten Messdaten vom Langzeitmonitoring archiviert und weiterverarbeitet 
werden. Dem medizinischen Personal soll somit neben morphologischen Bildaufnahmen eine unterstützende, nichtinvasive Diagnostik zur Verfügung stehen und der Patient die Möglichkeit eines dokumentierten Heilungsverlaufes erhalten.

\section{Einleitung}

Zurzeit wird jährlich in Deutschland ambulant und stationär mit Hilfe des in der Medizin angewandten Steifverbandes eine Ruhigstellung bei über 200.000 Unterarmfrakturen (Ulna- und Radiusfraktur), nach einer Reposition der Fragmente, oder nach operativer Behandlung von Sehnen- und Bänderrissen gewährleistet. Durch das in der Regel 6-wöchige Tragen kommt es zu einer Beeinträchtigung der Nerven und Muskelfunktion und durch den Sauerstoffabschluss entsteht ein feuchtes Milieu. Dieses führt bei offenen Wunden zu Infektionen, Wundheilstörungen und zu überschießender Narbenbildung. Neben dem traditionellem Gipsverband und dem synthetischen Castverband findet immer mehr die wiederverwendbare, gipsstabile Handgelenksorthese Anwendung. Diese gewährleisten neben der kompletten Schienung der Extremität, wie in Abbildung 2 dargestellt, auch die kontrollierte Beweglichkeit in dorsalflexion und palmarflexion durch ein modulares Gelenk für eine frühfunktionelle Therapie.
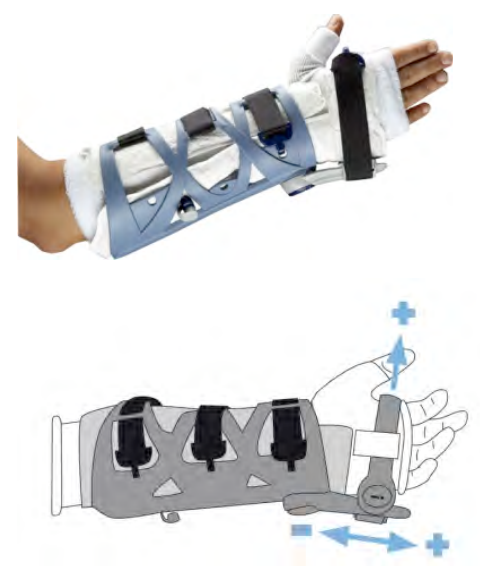

Abb. 2: Darstellung der gipsstabilen Handgelenksorthese mit modularem Gelenk

Bei den Therapien stehen während der Heilung meist morphologische Bildaufnahmen in Form von Röntgen- oder CT-Aufnahmen zur Verfügung. Komplikationen bei konservativer wie auch operativer Therapie können auftreten. Eine frühzeitige und intelligente Erkennung von Druckstellen, Durchblutungsstörungen wie auch das Erfassen von Entzündungsindikatoren in Form von Rötungen, Überwärmung und Schwellungen für einen komplikationsfreien Heilungsverlauf sind nicht gegeben. Erst das Auftreten von Schmerzen oder die Beeinträchtigung von Funktion der gesamten Extremität lassen auf Komplikationen schließen. Diese werden erst spät vom Patienten wahrgenommen und gefährden bzw. verzögern einen optimalen Heilungsprozess. Trophische Erkrankungen wie z.B. beim komplexen regionalen Schmerzsyndrom (CRPS), die sich auf Grund von Stößen oder einer Fraktur ergeben können, werden nicht ohne weiteres erkannt. So werden Temperaturschwankungen von bis $\mathrm{zu} 2{ }^{\circ} \mathrm{C}$ im betroffenen Körperglied und ein Anstieg von Schweißsekret als Reflexmuster bei einem akuten CRPS angenommen und stellen eine gestörte Thermoregulation da. Erst eine Langzeitmessung der Hauttemperatur und deren Schwankungen des betroffenen Körpergliedes und des kontralateralen Körpergliedes ermöglichten eine Parametrisierung des CRPS (vgl. KRUMOVA et al., 2008). 


\section{Methoden}

Mit dem entwickeltem Embedded-System wird drahtlos an inguinalen und axillaren Messpunkten die Körpertemperatur des Patienten gemessen. Diese liegt annähernd bei 1-2 ${ }^{\circ} \mathrm{C}$ unter der eigentlichen Kerntemperatur (Chaturvedi et al., 2004;Insler et al., 2006). Durch das textilbasierte Multi-Sensorsystem wird zum einen eine großflächige Temperaturverteilung der betroffenen Extremität durch ein SensorArray erfasst, was eine thermographische Bildgebung über einen längeren Zeitraum ermöglicht. Zum anderen wird die herrschende Hautfeuchtigkeit in der Handgelenksorthese gemessen. Dies soll eine frühzeitige Erkennung von Endzündungsmustern ermöglichen. Die Bewegungen der betroffenen Extremität wird mittels integriertem Gyroskop kontinuierlich gemessen, so dass der behandelnde Facharzt und Therapeut die aufgezeichnete Beanspruchung auswerten und bei Bedarf entsprechende Maßnahmen ergreifen kann.

\section{Embedded-System und Sensoren}

Zur Erfassung, Speicherung, Auswertung der Sensordaten und für die drahtlose Kommunikation über Bluetooth 2.1+EDR sowie über eine IR-Schnittstelle, wird ein Embedded-Board mit einem AVR-RISCMikrocontroller vom Typ ATmega128 verwendet. Dieses ist in einem desinfizierbaren und schlagfesten ABS-Miniaturgehäuse samt Akkupack untergebracht. Um den zeitlichen Verlauf der Messungen zu Erfassen werden mit Hilfe einer Real-Time-Clock (RTC) die Messdaten mit einem Zeitstempel versehen.

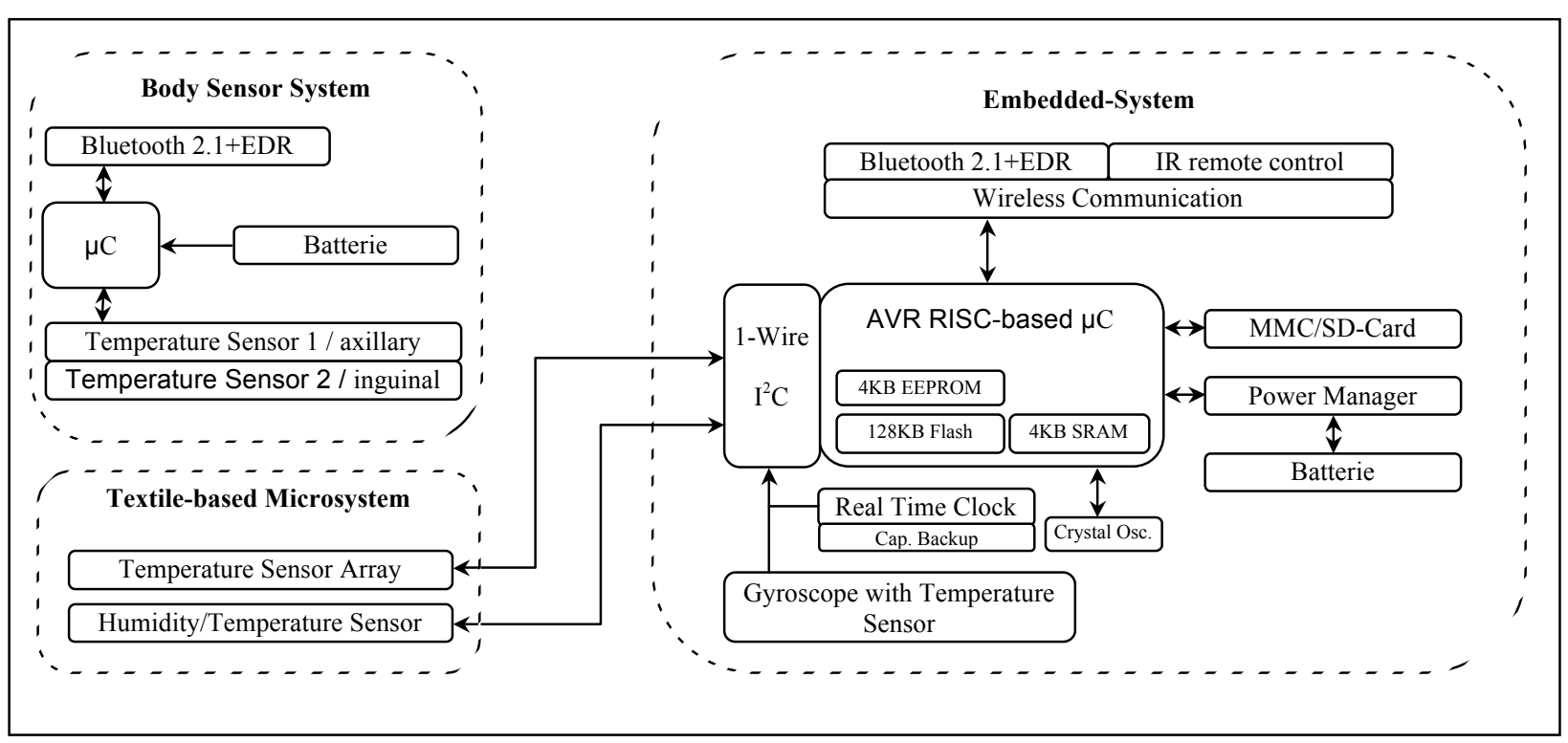

Abb. 3: Schematische Darstellung der Teilkomponenten des Embedded-Systems mit Sensoranbindung

Durch einen Pufferkondensator ist ein sicherer Betrieb der RTC weit über 100 Tage gewährleistet. Für die Erfassung der wirkenden Belastung auf die gipsstabile Handgelenksorthese ist ein digitales 3-Achsen MEMS Gyroskop integriert. Dieser verfügt über drei 16-Bit Analog-to-Digital-Konverter (ADCs) zur simultanen Abtastung der Gyroskopwerte ohne externen Multiplexer. Neben einem internen Tiefpassfilter mit programmierbarer Bandbreite steht eine Fast-Mode $I^{2} \mathrm{C}$ Schnittstelle für die Kommunikation mit dem Embedded-System zur Verfügung. Zudem verfügt das hermetisch versiegelte und auf Wafer-Ebene 
gebondete Gyroskop über einen weiteren Temperatursensor mit digitalem Ausgang, der für die Erfassung der Umgebungstemperatur genutzt wird.

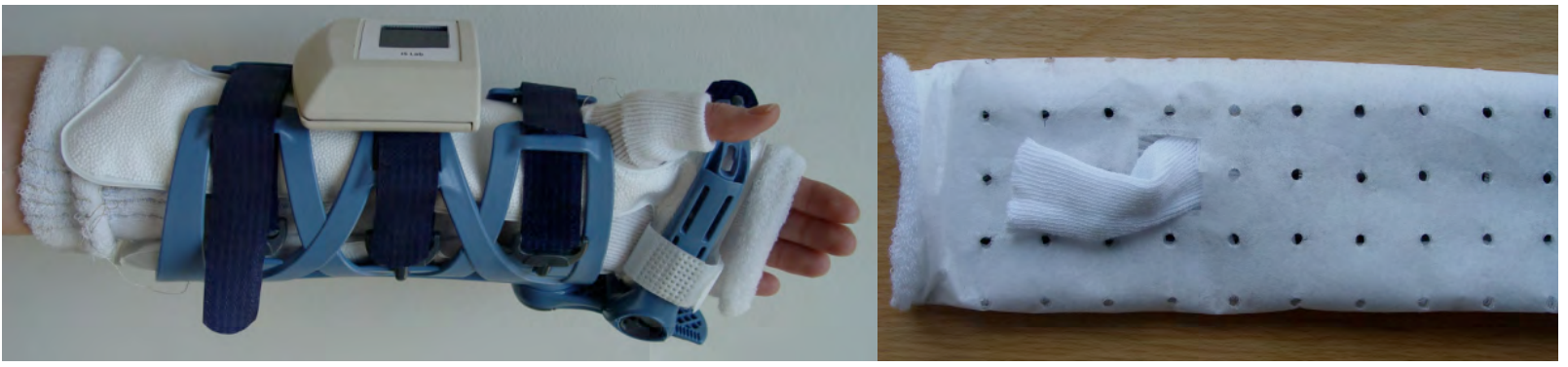

Abb. 4: Embedded-System und das in die Handgelenksorthese integrierte textilbasierte Mikrosystem

Das textilbasierte Mikrosystem besteht neben dem Luftfeuchtigkeitssensor, welcher über den $I^{2} \mathrm{C}$-BUS angesprochen wird, aus einem 10x10 großen Temperatursensor-Array, welches in den Frotteebezug der Orthese eingenäht ist. Hierzu wurde mit Silberpartikel bestücktes Fadenmaterial in die Textilstruktur eingenäht und die Sensoren zu einer Busstruktur verlötet. Die vorhandenen digitalen Temperatursensoren verfügen über eine 64-Bit Adressierung und können so einzeln über den 1-WireBUS angebrochen und ausgewertet werden. Die Grundgenauigkeit der Sensoren beträgt $\pm 0.5{ }^{\circ} \mathrm{C}$ bei einer Auflösung von 9-Bit. Mit Hilfe einer Registererweiterung auf 12-Bit des sensoreigenen Mikrocontrollers und einer Umrechnung der Messwerte im Embedded-System kann rechnerische die Auflösung auf $0.0625^{\circ} \mathrm{C}$ erhöht werden. Für die sichere Langzeitspeicherung steht im Embedded-System eine 2 GB große SD-Karte zu Verfügung. 


\section{Bildverarbeitung}

Um eine visuelle Darstellung der beteiligten Extremität und dessen Temperaturverlauf mit Hilfe des 10x10 Temperatursensor-Array zu erstellen, müssen zu den bekannten Sensorkoordinaten zusätzliche farbige Pixel berechnet werden. Mit Hilfe der Interpolation ist dies möglich. Die „Nearest Neighbor“ Interpolation stellt einen sehr einfachen Algorithmus dar. Hier besteht ein Raster aus den bekannten Koordinaten der einzelnen Temperatursensoren. Ein neuer Pixel bekommt die Farbe des Nachbarpixels was, wie in Abbildung 5 dargestellt, zu einem "pixeligen" Erscheinungsbild der Extremitäten führt.

$$
f(x, y)=\left\{\begin{array}{l}
f\left(Q_{1 \mathbf{1}}\right), x<x_{\mathbf{2}}-\frac{\boldsymbol{\Delta} x}{2}, y<y_{\mathbf{2}}-\frac{\boldsymbol{\Delta} y}{2} \\
f\left(Q_{1 \mathbf{2}}\right), x>x_{\mathbf{2}}-\frac{\boldsymbol{\Delta} x}{\mathbf{2}}, y<y_{\mathbf{2}}-\frac{\boldsymbol{\Delta} y}{2} \\
f\left(Q_{2 \mathbf{1}}\right), x<x_{\mathbf{2}}-\frac{\boldsymbol{\Delta} x}{2}, y>y_{\mathbf{2}}-\frac{\boldsymbol{\Delta} y}{\mathbf{2}} \\
f\left(Q_{2 \mathbf{2}}\right), x>x_{\mathbf{2}}-\frac{\boldsymbol{\Delta} x}{\mathbf{2}}, y>y_{\mathbf{2}}-\frac{\boldsymbol{\Delta} y}{\mathbf{2}}
\end{array}\right.
$$
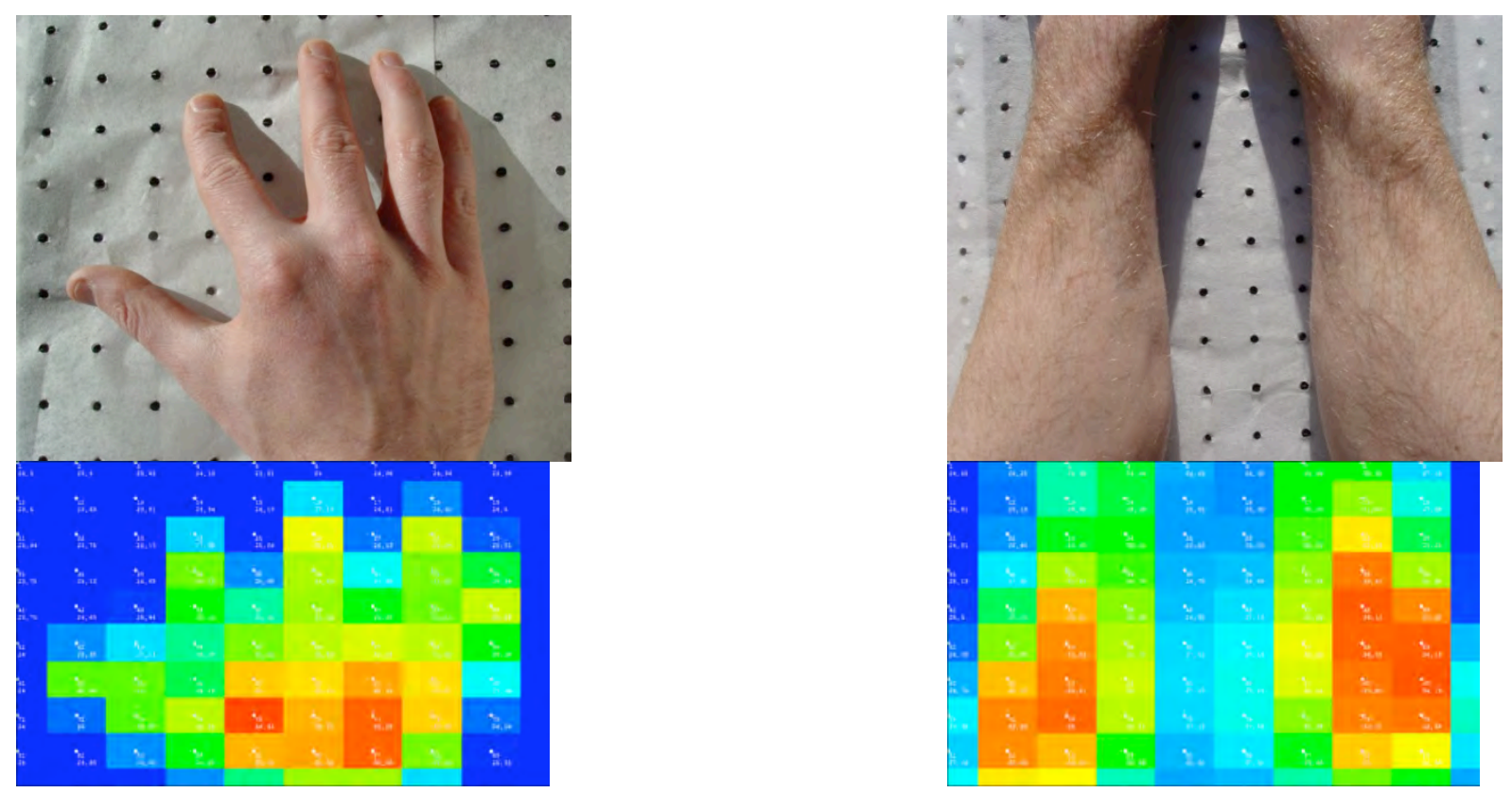

Abb. 5: Darstellung der Extremität und Ergebnis der „Nearest Neighbor“-Interpolation.

Im Verlauf der Interpolation wird eine Wandlung der Temperaturwerte in RGB-Farbraum vollzogen. Hierzu werden alle Werte $k$ aus einem Intervall von $\left[0,1024\left[\right.\right.$ nach $\mathbf{R}^{\mathbf{z}} \mid[0,255]$ abgebildet. Hieraus entsteht folgende Farbzuweisung:

$$
c=\left\{\begin{array}{rc}
(0, k, 255), & k<256 \\
(0,255,512-k), & 256 \leq k<512 \\
(k-512,255,0), & 512 \leq k<768 \\
(255,1024-k, 0), & 768 \leq k<1024,
\end{array}\right.
$$

Die Variable c bildet die Farbe im RGB-Farbraum, $R \in[0,255], G \in[0,255], B \in[0,255]$ ab. 
Eine weitere Möglichkeit stellt die bilineare Interpolation dar, bei der zwischen den gegebenen Koordinaten eine lineare Interpolation in $x$ - als auch in y-Richtung durchgeführt wird. Der gesuchte Wert liegt zwischen vier definierten Rasterpunkten. Bei diesem Verfahren weist das Erscheinungsbild der Extremitäten, wie in Abbildung 6 gezeigt, Stufen auf. Die Konturen sind aber besser zu erkennen als bei der „Nearest Neighbor“ Interpolation.

$$
\begin{aligned}
& f(x, y) \approx \frac{f\left(Q_{11}\right)}{\left(x_{2}-x_{1}\right)\left(y_{2}-y_{1}\right)}\left(x_{2}-x\right)\left(y_{2}-y\right) \\
& +\frac{f\left(Q_{21}\right)}{\left(x_{2}-x_{1}\right)\left(y_{2}-y_{1}\right)}\left(x-x_{1}\right)\left(y_{2}-y\right) \\
& +\frac{f\left(Q_{12}\right)}{\left(x_{2}-x_{1}\right)\left(y_{2}-y_{1}\right)}\left(x_{2}-x\right)\left(\boldsymbol{y}-y_{1}\right) \\
& +\frac{f\left(Q_{22}\right)}{\left(x_{2}-x_{1}\right)\left(y_{2}-y_{1}\right)}\left(x-x_{1}\right)\left(y-y_{1}\right)
\end{aligned}
$$
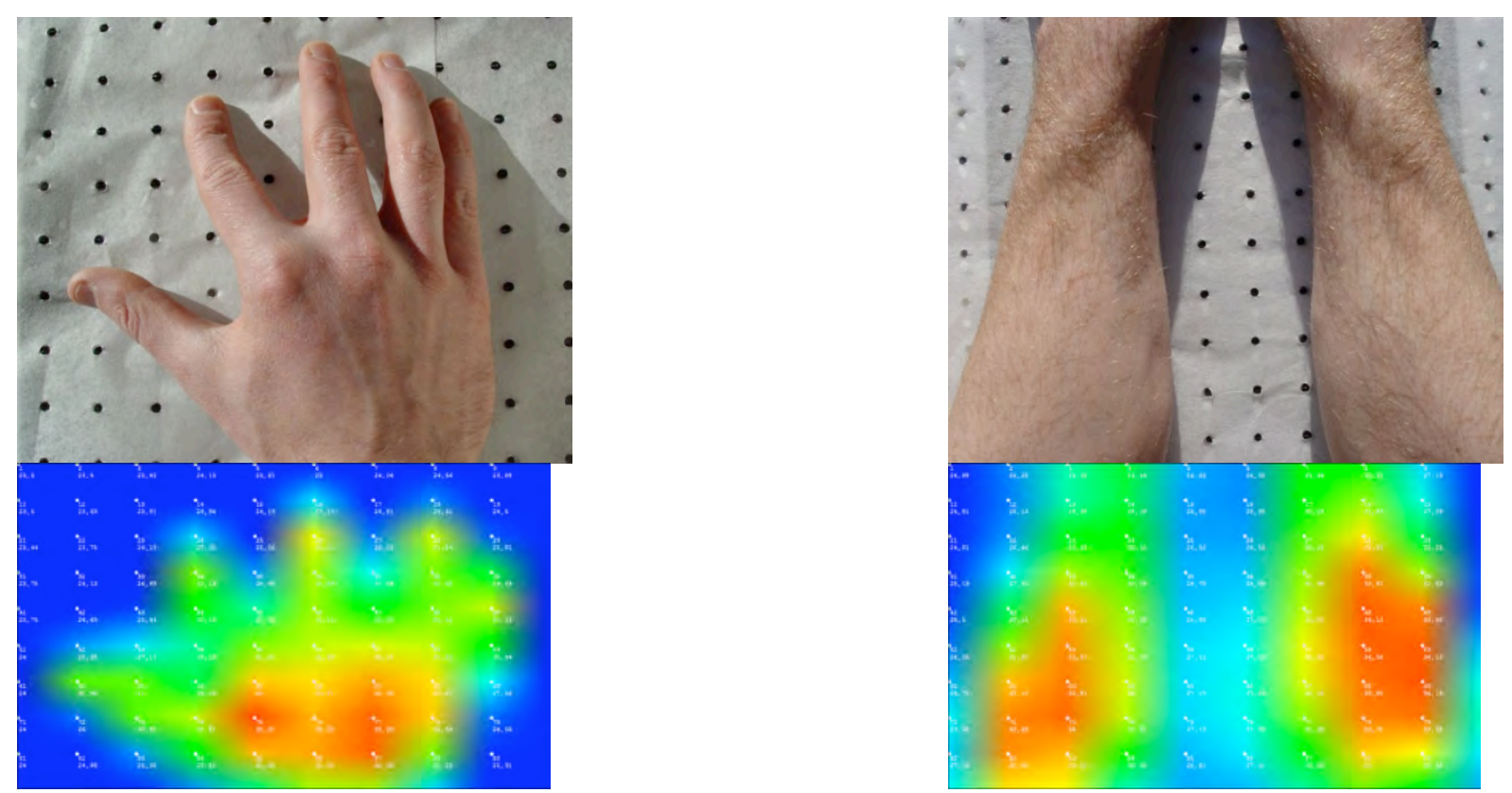

Abb. 6: Darstellung der Extremität und Ergebnis der bilinearen Interpolation.

Bei der bikubischen Spline-Interpolation werden die Werte von mehreren benachbarten Pixel erfasst und daraus interpolierte Werte berechnet. Zu Ermittlung der Koeffizienten $a_{i j}$, der auf die Messdaten passenden Splinefunkion, werden bekannte Koordinaten von 16 benachbarten Punkten an eine Polynomfunktion 3. Grades angepasst. Daraus entsteht die sogenannte B-Spline-Kurve. Die Funktionswerte der ermittelten Splinefunktion können anschließend als interpolierte Werte genutzt werden.

Bei diesem Verfahren weist das Erscheinungsbild der Extremitäten keine Stufen auf, die Konturen haben einen weichen Übergang und sind besser zu erkennen als bei den vorrangegangenen Interpolationen. 

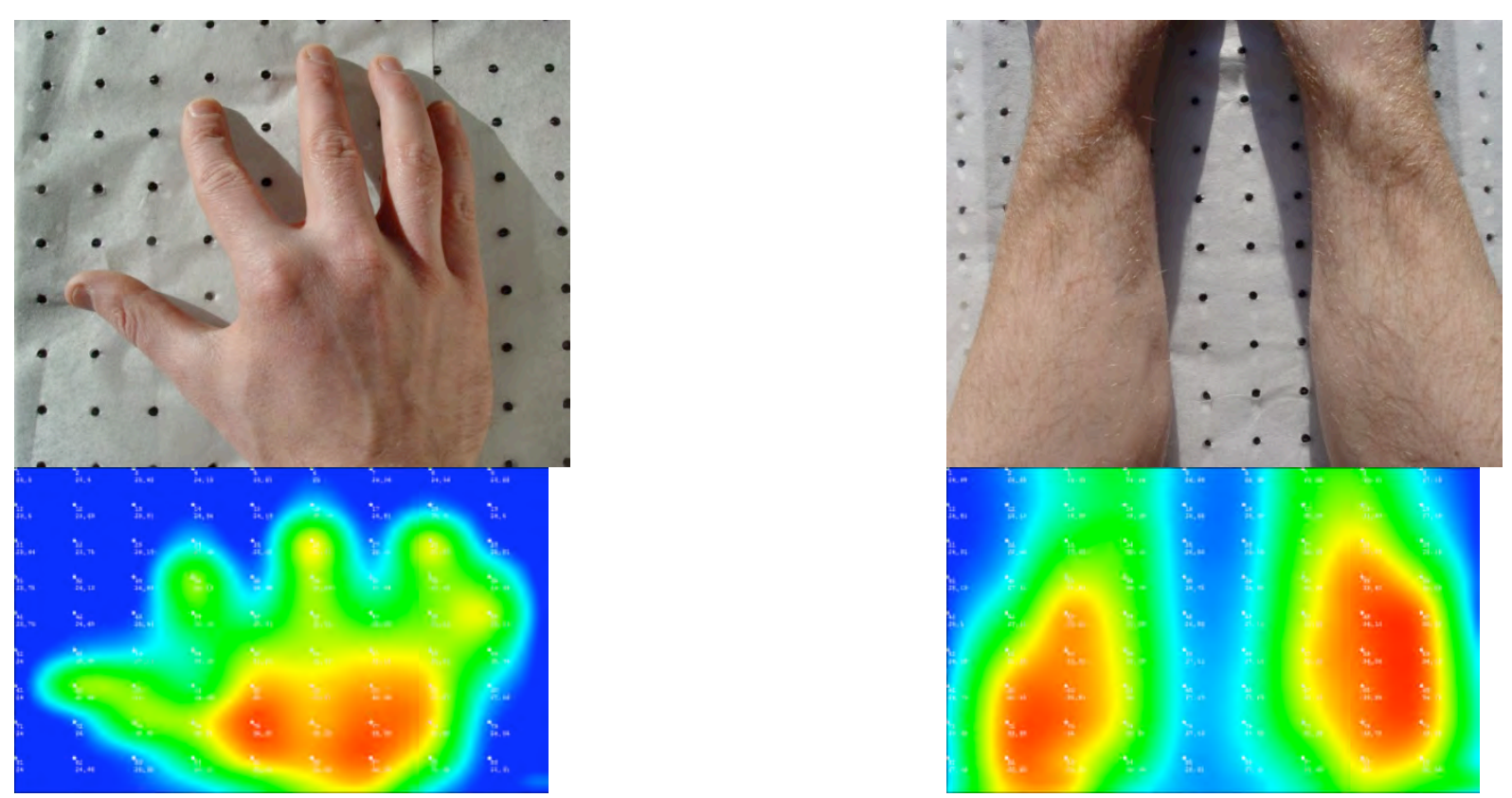

Abb. 7: Darstellung der Extremität und Ergebnis der bikubischen Interpolation.

\section{Ergebnisse}

Im Rahmen des Forschungsprojektes konnte gezeigt werden, dass es möglich ist ein bildgebendes, textilbasiertes Mikrosystem zu realisieren, welches in eine gipsstabile Handgelenksorthese integriert werden kann. Die Anwendungsgebiete für solch ein Mikrosystem lassen sich schnell erweitern. Hier werden in Zukunft Zuverlässigkeitsuntersuchungen in Form von Waschversuchen bzw. die Anwendung des Autoklaven zur Desinfektion eine große Rolle spielen. Die drahtlose Erfassung der Körpertemperatur am inguinalen und axillaren Messpunkt zeigte sich als problemlos. Potenzial bietet das Energiemanagement der Bluetooth-Module wie auch des Embedded-Systems selbst. Auch die kontinuierliche Messwerterfassung des integrierten Gyroskops verläuft reibungslos. Mit Blick auf das Fadenmaterial könnte die webtechnische Herstellung des Sensor-Arrays eine Lösung bieten. Auch die Auswirkungen einer stark schwankenden Außentemperatur wird mehr in den Vordergrund rücken um im weiteren Verlauf eine angestrebte Mustererkennung zu realisieren.

\section{Literatur}

Chaturvedi D, Vilhekar KY, Chaturvedi P, Bharambe MS. Comparison of axillary temperature with rectal or oral temperature and determination of optimum placement time in children. Indian Pediatr 2004, 41: 600-603.

Insler SR, Sessler DI. Perioperative thermoregulation and temperature monitoring. Anesthesiol Clin 2006; 24: 832-837.

Krumova EK, Frettlöh J, Klauenberg S, Richter H, Wasner G, Maier C. Long-term skin temperature measurements - a practical diagnostic tool in Complex Regional Pain Syndrome. Pain 2008 Aug 22. 